\title{
Prediction of Glycogen and Moisture Contents in Japanese Wagyu Beef by Fourier Transform Near-infrared Spectroscopy for Quality Evaluation
}

\author{
Zhifeng Yao, ${ }^{1}$ Toshiaki Oe, ${ }^{4}$ Xiao Ye, ${ }^{1}$ Rui Yatabe, ${ }^{1,2}$ \\ Yusuke Tahara, ${ }^{2}$ Takeshi Onodera, ${ }^{1,2^{*}}$ Kiyoshi Toko, ${ }^{2,3}$ \\ Masami Nishimura, ${ }^{4}$ Ken Iwao, ${ }^{4}$ and Naruhiko Tanaka ${ }^{4}$ \\ ${ }^{1}$ Graduate School of Information Science and Electrical Engineering, Kyushu University, \\ 744 Motooka, Nishi Ward, Fukuoka 819-0395, Japan \\ ${ }^{2}$ Research and Development Center for Five-Sense Devices, Kyushu University, \\ 744 Motooka, Nishi Ward, Fukuoka 819-0395, Japan \\ ${ }^{3}$ Institute for Advanced Study, Kyushu University, \\ 744 Motooka, Nishi Ward, Fukuoka 819-0395, Japan \\ ${ }^{4}$ Tottori Prefectural Animal Husbandry Experiment Station, \\ 606 Matsudani, Kotoura, Touhaku, Tottori 689-2503, Japan
}

(Received March 15, 2019; accepted June 19, 2019)

Keywords: glycogen, moisture content, Japanese wagyu beef, FT-NIR, PLSR

Glycogen and moisture contents are associated with palatability, especially the flavor of Japanese wagyu beef samples. In this study, near-infrared (NIR) absorbance spectroscopy $\left(12500-4000 \mathrm{~cm}^{-1}\right.$ ) was carried out to predict the glycogen and moisture contents in Japanese wagyu beef. Calibration and prediction models were established by partial least-squares regression (PLSR) between the measured reference glycogen and moisture contents and the spectral data. Different spectral preprocessing methods were used, and the loading coefficient obtained from PLSR models was employed to select feature spectra. As a result, the prediction model of glycogen with the selected spectral region of 9000-4300 $\mathrm{cm}^{-1}$ after smoothingstandard normalized variate (SNV) yielded optimum results with a determination coefficient $\left(R_{p}{ }^{2}\right)$ of 0.415 , a root-mean-squared error of prediction (RMSEP) set of $0.386 \mathrm{mg} / \mathrm{g}$, and a ratio of prediction to deviation (RPD) of 1.218. In addition, the prediction model of moisture content in the full spectral region of $12500-4000 \mathrm{~cm}^{-1}$ after smoothing-multiplicative scatter correction (MSC) yielded optimum results with $R_{p}{ }^{2}$ of 0.795 , RMSEP of $2.669 \%$, and RPD of 2.008 . The results of this study demonstrated that NIR spectroscopy offers great potential for the prediction of glycogen and moisture contents in Japanese wagyu beef samples.

\section{Introduction}

The palatability of beef can be attributed to three primary factors, namely, tenderness, juiciness, and flavor. ${ }^{(1)}$ In Japan, the beef marbling standard plays a critical role in evaluating

*Corresponding author: e-mail: onodera@ed.kyushu-u.ac.jp

https://doi.org/10.18494/SAM.2019.2372 
meat quality. ${ }^{(2)}$ Several researchers have shown that high marbling levels have a positive effect on Japanese consumers' preference owing to the improvement in beef quality such as tenderness. $^{(2-7)}$ This is consistent with the conclusion that tenderness is the most important factor affecting beef palatability. ${ }^{(8-11)}$ However, additional studies have shown that when tenderness is at an acceptable level, flavor becomes the next most important factor affecting the beef preference of consumers. ${ }^{(10-15)}$

Flavor is a complex concept, which is composed of taste, odor, and the interaction of these factors. In our previous work, a sensory panel test was used to determine the relationship between sensory attributes (e.g., sweetness, fattiness, aroma, tenderness, flavor, and overall evaluation) and chemical composition characteristics (e.g., moisture, protein, free amino acid, glycogen, and fatty acid contents) of Japanese wagyu beef by the simmering method. ${ }^{(16)}$ We demonstrated that the 'flavor' of sensory attributes was associated with glycogen and moisture contents. It has been widely reported that pre rigor muscle glycogen content has a negative curvilinear relationship with ultimate $\mathrm{pH},{ }^{(17)}$ which is an indicator of the final palatability of meat. ${ }^{(18,19)}$ In addition, the glycogen content in the muscle is related to the amounts of monosaccharides such as glucose and fructose after slaughter, which affect the development of meat flavor via the Maillard reaction; therefore, glycogen contributes to beef palatability. ${ }^{(20)}$ Moisture content is often used to assess meat quality. ${ }^{(21)}$ It also has a strong relationship with the contents of lipids that affect sensory attributes such as flavor, juiciness, texture, and appearance. ${ }^{(22)}$ Therefore, to satisfy an increasing demand for evaluating meat quality by either the meat processing industry or consumers, fast, accurate, and nondestructive methods are urgently required.

In recent years, near-infrared (NIR) spectroscopy, which is based on optical measurements of reflectance, transmittance, and interactance, has been considered as a nondestructive, fast, convenient, inexpensive, and precise technique. NIR spectroscopy combined with various multivariable data processing techniques has been effectively employed. Applications of the NIR technique to the evaluation of meat quality have been reviewed. ${ }^{(23)}$ The NIR technique has the potential of detecting meat adulteration, ${ }^{(24,25)}$ assessing meat microbial spoilage, ${ }^{(26,27)}$ and estimating meat quality. ${ }^{(28,29)}$

Nowadays, several research studies on Japanese wagyu beef have shown that NIR spectroscopy can be applied to predict the fat content and quantify the fatty acid composition. Kobayashi et al. have demonstrated the correlation between fat content and spectral information in Japanese raw beef. ${ }^{(30)}$ Piao et al. predicted the monounsaturated fatty acid (MUFA), oleic acid, and saturated fatty acid (SFA) compositions in Japanese Black cattle with the coefficients of determination of $0.69,0.64$, and 0.67 , respectively. ${ }^{(31)}$ The reason why many researchers focus on the fat in Japanese wagyu beef is that fat is an important factor that affects the overall palatability and taste. However, according to Suzuki et al., in Japanese Black cattle, the chemical composition characteristics, such as the amounts of sugar (e.g., glycogen, glucose, and ribose) and ATP-related compounds, are related to the 'overall evaluation', and at the same time, the amounts of ATP-related compounds correlate significantly with the 'umami intensity'.(32) The conclusion that the content and composition of fat are not the only indexes for the taste of Japanese Black cattle was suggested. Our previous work where glycogen and moisture contents 
affected the flavor and tenderness has a similar conclusion. ${ }^{(16)}$ In addition, to the best of our knowledge, there has been little research so far on the measurement of glycogen and moisture contents for the quality evaluation of Japanese wagyu beef based on NIR spectroscopy.

Therefore, the main objective of this study was to investigate the potential of using NIR spectroscopy and multivariable data processing methods for the rapid analysis of glycogen and moisture contents in Japanese wagyu beef for quality evaluation. The specific objectives are as follows: (1) to obtain spectral information of Japanese wagyu beef in the $12500-4000 \mathrm{~cm}^{-1}$ region, (2) to establish calibration and prediction models based on spectral data and measured glycogen and moisture contents based on partial least-squares regression (PLSR), and (3) to select the feature spectra linked to glycogen and moisture contents and evaluate the accuracy of prediction models using these feature spectra.

\section{Materials and Methods}

\subsection{Sample preparation}

The Japanese wagyu beef samples analyzed in this study were obtained from Tottori Prefecture, Japan. Carcass characteristics of meat located between the sixth and seventh ribs (the longissimus thoracis muscle) were evaluated following the standards of the Japan Meat Grading Association, ${ }^{(33)}$ and the quality grades of meat were higher than Grade 4 (4 or 5).

Twenty-four carcasses were aged at $0{ }^{\circ} \mathrm{C}$ for 19 days. One meat sample of the longissimus thoracis muscle was collected from each of the 24 carcasses, minced, and stored at $-30{ }^{\circ} \mathrm{C}$ until analysis. Each meat sample has an identification number. Before the chemical analyses of glycogen and moisture contents and the measurement by FT-NIR, the samples were thawed at $4{ }^{\circ} \mathrm{C}$ for $4 \mathrm{~h}$.

\subsection{Determination of glycogen and moisture contents}

The glycogen content was determined by the iodine binding method according to Dreiling et al. ${ }^{(34)}$ About $0.5 \mathrm{~g}$ of meat sample was homogenized with $5 \mathrm{ml}$ of cold $7 \%$ perchloric acid for $30 \mathrm{~s}$ at $30000 \mathrm{rpm}$. Samples were subsequently left for $10 \mathrm{~min}$ at room temperature and then filtered with filter paper of $55 \mu \mathrm{m}$ pore size. The fraction including glycogen was collected and used for glycogen measurement. The glycogen content was determined using a spectrophotometer (Shimadzu, UV-1200V, Japan) at $460 \mathrm{~nm}$ and calculated as milligrams per gram of raw meat.

Moisture content was measured using a ProFat system (CEM, ProFat TM Raw Meat Fat Analyzer, USA) according to the method of the Association of Official Analytical Chemists (AOAC, 2008.06). The system was developed to combine microwave drying with nuclear magnetic resonance. A $1.5 \mathrm{~g}$ sample was weighed in the system, where its moisture content was analyzed by determining the weight loss. Moisture content was calculated as $\mathrm{w} / \mathrm{w} \%$. 


\subsection{NIR spectroscopy measurements}

Prior to acquiring the spectra, a $3 \mathrm{~g}$ sample was weighed. NIR spectra were collected using a FT-NIR spectrometer (PerkinElmer, Frontier NIR Spectrometer, USA), equipped with an NIR reflection accessory (PerkinElmer, NIRA, USA) for diffuse reflectance measurements. The samples were directly measured by diffuse reflectance without any preparation at ambient temperature. Each spectrum was the average of 16 scans at $8 \mathrm{~cm}^{-1}$ resolution over the range of 12500 to $4000 \mathrm{~cm}^{-1}$. The absorbance spectra were acquired as logarithmically transformed reflectance $\log (1 / R)$.

\subsection{Data analysis}

\subsubsection{Spectral preprocessing}

To reduce sample-to-sample variability due to scatter and optical interference and improve the predictive capability of models, various mathematical preprocessing techniques including Savitzky-Golay (SG) smoothing, multiplicative scatter correction (MSC), and standard normalized variate (SNV) were used before data modeling. The SG smoothing method can remove high-frequency noise, smooth data, and improve the signal-to-noise ratio of the spectral curve while retaining important information. ${ }^{(35)}$ MSC is adopted as a widely used spectral preprocessing technique that can eliminate direct reflection and noise in the diffuse reflection and separate multiplicative interferences such as scatter and particle size at the same time. ${ }^{(36)}$ Identically, SNV is used to correct baseline drift and eliminate undesirable scattering effects from the data matrix. ${ }^{(37)}$

To optimize the accuracy of models, in this work, the spectral preprocessing methods performed were nontreatment, MSC, SNV, and SG smoothing with a second-order polynomial and window sizes of 23 points, as well as combinations of two preprocessing methods. Then, the preprocessed spectra were used for modelling.

\subsubsection{Multivariable data modelling}

Outliers are samples not fitting in the model. Outlier detection is important before modelling because even a single outlier may affect the robustness of the model and lead to incorrect conclusions. In this study, principal component analysis (PCA) was performed to identify and, if necessary, eliminate spectral outliers using Hotelling's $T^{2}$ statistics with a $95 \%$ confidence threshold. ${ }^{(38)}$

PLSR models, as a widely used and highly effective analysis, are developed between the relation preprocessed spectra ( $X$-matrix) and physical and chemical parameters ( $Y$-matrix). The procedure of PLSR modelling has 2 steps. The first is the development of calibration models, which are established on the basis of calibration data. To ensure that the calibration models obtained are not overfitting, a cross validation using the leave-one-out method is considered. The number of latent variables (LVs) to be selected in PLSR models can also be determined 
by the above cross validation. ${ }^{(39)}$ The second is the development of prediction models based on validation methods. Prediction models are used to examine the reliability of the calibration models and ensure the related calibration models can work. Two types of validation methods are widely used for developing prediction models. One is test-set validation, where totally new and external data will be used for prediction. The other is cross validation, where part of the calibration data will be used for prediction. The common methods of cross validation are full cross-validation and leave-one-out cross validation. In this study, owing to the restricted number of samples, after calibration models were developed, the leave-one-out cross validation was simultaneously used for the validation of calibration models and the establishment of prediction models of glycogen and moisture contents.

The performance characteristics of PLSR models were evaluated in terms of the determination coefficients for calibration $\left(R_{c}{ }^{2}\right)$ and prediction $\left(R_{p}{ }^{2}\right)$, the root-mean-squared errors of calibration (RMSEC) and prediction (RMSEP), and the ratio of prediction to deviation (RPD). RPD is calculated using the ratio of standard deviation (SD) of the reference values over the RMSEP. Generally, good models should have higher $R_{c}{ }^{2}$ and $R_{p}{ }^{2}$ values and lower RMSEC and RMSEP values. The small difference between RMSEC and RMSEP indicates the robustness of the models. In addition, an RPD value above 2 is considered to be a reasonably good prediction, and greater than 3 means an excellent prediction. ${ }^{(40)}$

\subsubsection{Feature spectral selection}

Feature spectral selection is important for quantitative analysis because it eliminates irrelevant wavelengths and selects the informative ones for effective analysis. In this study, loading coefficients in the PLSR model were used to select the wavelengths that most highly affect glycogen and moisture content prediction in Japanese wagyu beef. In general, the loading coefficient has a profile similar to the nontreated spectral data and may highlight regions of high importance. Using the feature spectra, new PLSR models were established. All data analysis procedures used throughout the work were implemented using the Unscrambler X 10.4 software (CAMO Software, X 10.5, Norway).

\section{Results and Discussion}

\subsection{Reference measurement of glycogen and moisture contents}

Glycogen as the main form of carbohydrate storage in animals is one of the important chemical parameters that determine meat palatability. ${ }^{(41,42)}$ A previous study of beef sensory evaluation demonstrated that a high glycogen content is associated with improved tenderness, bright red color, and enhanced flavor, ${ }^{(43)}$ therefore, glycogen content has a positive effect on beef palatability. Additionally, it is known that moisture is the most abundant component in muscle and is essential for beef quality. Previous research showed that the moisture content negatively correlated with the fat content and affected the texture of Japanese black steers. ${ }^{(2)}$ Because of its relationship with the contents of lipids, moisture content also affects the factors 
affecting palatability such as juiciness and flavor. ${ }^{(22)}$ The reference results of glycogen and moisture contents for Japanese wagyu beef analyzed in this study are presented in Table 1. The glycogen content of 24 samples ranged between 0.52 and $2.35 \mathrm{mg} / \mathrm{g}$, which were in good agreement with the results of Komatsu et al. ${ }^{(20)}$

\subsection{Spectral data analysis}

In the $12500-4000 \mathrm{~cm}^{-1}$ region, the original absorbance spectra of Japanese wagyu beef samples are shown in Fig. 1. In general, NIR spectra are sensitive to the fundamental vibrations of $\mathrm{C}-\mathrm{H}, \mathrm{O}-\mathrm{H}, \mathrm{S}-\mathrm{H}$, or $\mathrm{N}-\mathrm{H}$ bonds in organic compounds, and the absorption peaks are associated with the vibrations of these functional groups. ${ }^{(44)}$ According to a previous study, the peaks in the NIR region at approximately 6800 and $5100 \mathrm{~cm}^{-1}$ are probably related to the $\mathrm{O}-\mathrm{H}$ stretching in moisture. ${ }^{(45)}$ In addition, Brown reported that wavelengths located at $1420 \mathrm{~nm}\left(7040 \mathrm{~cm}^{-1}\right), 1580 \mathrm{~nm}\left(6330 \mathrm{~cm}^{-1}\right), 1750 \mathrm{~nm}\left(5710 \mathrm{~cm}^{-1}\right)$, and $1690 \mathrm{~nm}\left(5910 \mathrm{~cm}^{-1}\right)$ were associated with the absorption of glycogen when using visible-NIR spectroscopy for the quantitative analysis of oyster samples. ${ }^{(46)}$ In our work, only the absorption peaks of 5710 and $5910 \mathrm{~cm}^{-1}$ were observed. This may be because oyster samples have a higher glycogen content than beef samples.

To reduce the effects of sample preparation, particle size of samples, and light scatter, spectral preprocessing methods were performed. After preprocessing, new spectra with less variation and scatter effects were obtained. PCA was used on nontreated spectral data and preprocessing spectral data to detect outliers. Hotelling's $T^{2}$ values of each individual sample spectra were calculated to detect outliers using a $95 \%$ confidence threshold. The spectra of the same sample with nontreated and smoothing-treated methods were identified as the outlier and removed before PLSR modelling. It may be explained by the fact that the moisture content of the sample as the outlier was $30.97 \%$. Taking moisture content into consideration, this sample was also an outlier in a total of 24 beef samples.

Table 1

Reference measurement of glycogen and moisture contents of 24 Japanese wagyu beef samples.

\begin{tabular}{lrrrr}
\hline & Mean & SD & \multicolumn{1}{c}{ Min. } & Max. \\
\hline Glycogen (mg/g) & 1.16 & 0.47 & 0.52 & 2.35 \\
Moisture (w/w\%) & 40.95 & 5.36 & 30.97 & 51.64 \\
\hline
\end{tabular}

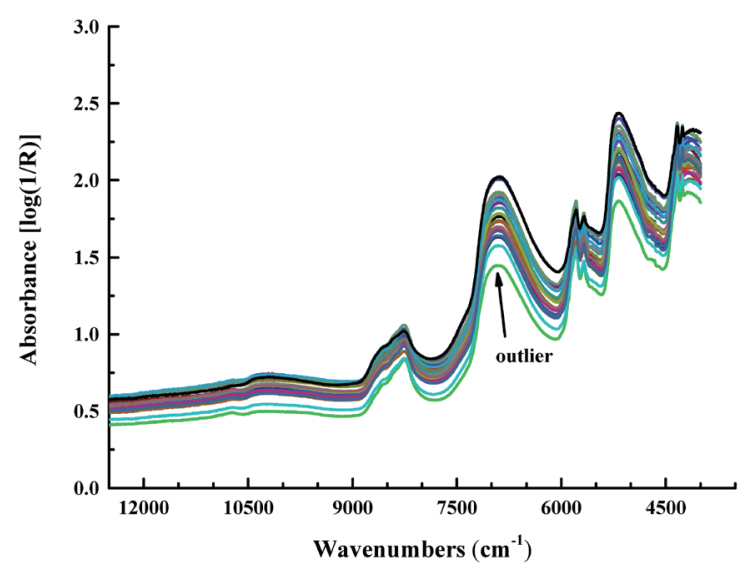

Fig. 1. (Color online) Nontreated spectra of 24 Japanese wagyu beef samples. Labelled spectra were identified as an outlier using Hotelling's $T^{2}$ statistics with a $95 \%$ confidence threshold. 


\subsection{Prediction of glycogen and moisture contents based on full spectra}

The prediction of glycogen and moisture contents was performed using PLSR models with nontreated spectra and the spectra preprocessed by different methods in the full spectral region of $12500-4000 \mathrm{~cm}^{-1}$. The results are shown at Table 2 .

The best PLSR model for glycogen prediction from 12500 to $4000 \mathrm{~cm}^{-1}$ was developed using smoothing-SNV-treated spectra. To visualize graphically the performance of this model, the measured reference and predicted values for calibration and prediction sets are shown in Fig. 2(a). It is shown that the best PLSR model for glycogen prediction has the highest $R_{p}{ }^{2}$ value of 0.403 , the lowest RMSEP value of $0.386 \mathrm{mg} / \mathrm{g}$, and an RPD value of 1.218 . The abovementioned results of this study with an $R_{p}{ }^{2}$ value of 0.403 in the prediction set were more

Table 2

Performance comparison of PLSR models for predicting glycogen and moisture contents based on full spectral data preprocessed by different methods.

\begin{tabular}{|c|c|c|c|c|c|c|c|c|}
\hline & \multirow{2}{*}{ Preprocessing } & \multirow{2}{*}{$\begin{array}{l}\text { Spectral region } \\
\left(\mathrm{cm}^{-1}\right)\end{array}$} & \multirow{2}{*}{ LV } & \multicolumn{2}{|c|}{ Calibration } & \multicolumn{2}{|c|}{ Prediction } & \multirow{2}{*}{ RPD } \\
\hline & & & & $R_{c}^{2}$ & RMSEC & $R_{p}^{2}$ & RMSEC & \\
\hline \multirow{6}{*}{$\begin{array}{l}\text { Glycogen } \\
(\mathrm{mg} / \mathrm{g})\end{array}$} & Nontreated & $12500-4000$ & 4 & 0.518 & 0.318 & 0.313 & 0.397 & 1.184 \\
\hline & Smoothing & $12500-4000$ & 4 & 0.518 & 0.318 & 0.321 & 0.388 & 1.211 \\
\hline & SNV & $12500-4000$ & 5 & 0.532 & 0.313 & 0.344 & 0.395 & 1.190 \\
\hline & MSC & $12500-4000$ & 2 & 0.475 & 0.332 & 0.386 & 0.386 & 1.218 \\
\hline & Smoothing-SNV & $12500-4000$ & 2 & 0.474 & 0.332 & 0.403 & 0.386 & 1.218 \\
\hline & Smoothing-MSC & $12500-4000$ & 2 & 0.530 & 0.314 & 0.237 & 0.399 & 1.178 \\
\hline \multirow{6}{*}{$\begin{array}{l}\text { Moisture } \\
(w / w \%)\end{array}$} & Nontreated & $12500-4000$ & 5 & 0.871 & 1.884 & 0.790 & 2.618 & 2.047 \\
\hline & Smoothing & $12500-4000$ & 5 & 0.871 & 1.888 & 0.758 & 2.568 & 2.087 \\
\hline & SNV & $12500-4000$ & 3 & 0.831 & 2.160 & 0.783 & 2.685 & 1.996 \\
\hline & MSC & $12500-4000$ & 3 & 0.831 & 2.163 & 0.751 & 2.713 & 1.976 \\
\hline & Smoothing-SNV & $12500-4000$ & 3 & 0.830 & 2.164 & 0.735 & 2.716 & 1.973 \\
\hline & Smoothing-MSC & $12500-4000$ & 3 & 0.830 & 2.166 & 0.795 & 2.669 & 2.008 \\
\hline
\end{tabular}

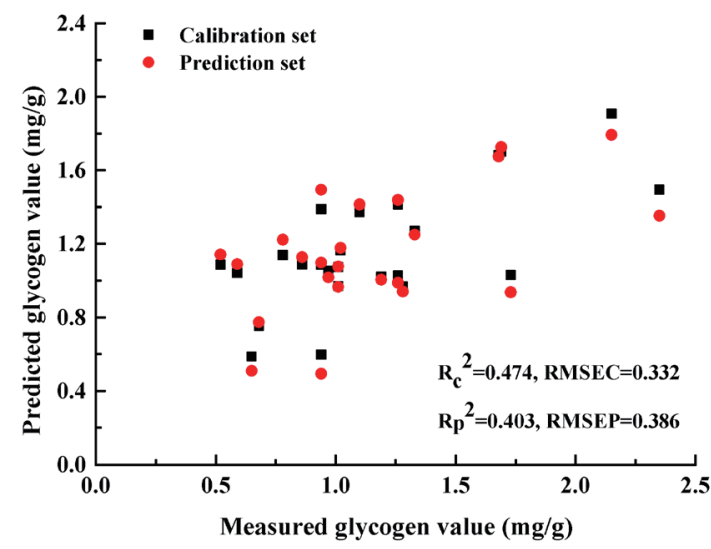

(a)

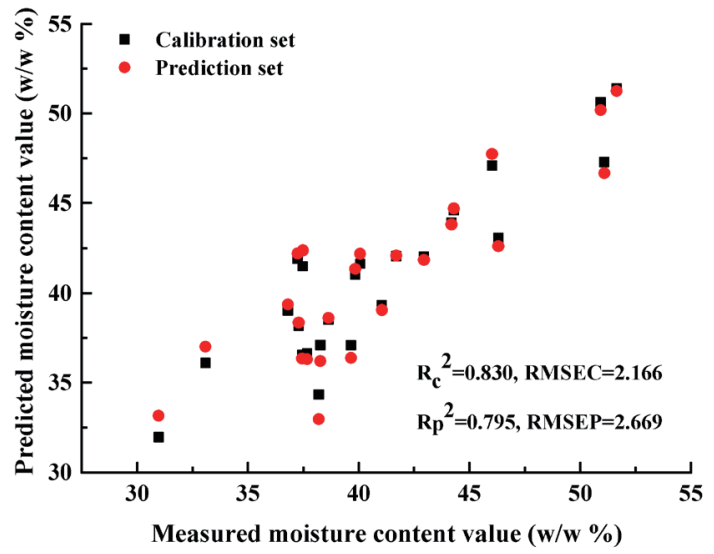

(b)

Fig. 2. (Color online) Best performance for predicting (a) glycogen and (b) moisture contents based on full spectra preprocessed by different methods. 
accurate than those of the previous study by Lomiwes et al. with an $R_{p}{ }^{2}$ value of 0.22 for the quantitative analysis of glycogen in beef samples. ${ }^{(47)}$ However, Rosenvold et al. measured 102 samples of beef $M$. longissimus lumborum using NIR spectroscopy and a prediction model, and an $R_{p}{ }^{2}$ value of 0.72 was obtained. ${ }^{(48)}$ The RPD value of the best PLSR model for glycogen content prediction was 1.218 , which also indicated that the model was not very robust. These results are partly due to the limited number of beef samples. Therefore, additional research studies with more samples and more scanning points in the same beef sample are needed to decrease the sample variability, thereby improving the model performance.

The performance characteristics of PLSR models for moisture content prediction based on sprectra from 12500 to $4000 \mathrm{~cm}^{-1}$ preprocessed by different methods were very similar. The preprocessing methods SNV and MSC produced similar results, mainly due to the correction of the baseline drift and the decrease in scattering for nontreated spectra. The best PLSR model for predicting moisture content was developed using smoothing-MSC-treated spectra and gave results with $R_{p}{ }^{2}$ of 0.795 , RMSEC of $2.166 \%$, and RMSEP of $2.669 \%$, as shown in Fig. 2(b). The RPD value was 2.008 , meaning that the model provided a reasonably good prediction. The results indicated that the prediction of moisture content by FT-NIR spectroscopy is feasible.

\subsection{Prediction of glycogen and moisture contents based on feature spectra}

In an NIR spectrum, there are large numbers of overtones and combination bands. To reduce the amount of input data, it is important to obtain the feature spectrum. In this study, the loading coefficient resulting from PLSR models was employed to select important spectra aiming to establish simplified PLSR models. As an example, for predicting the glycogen content of Japanese wagyu beef samples using nontreated spectra in the $12500-4000 \mathrm{~cm}^{-1}$ region, the relationship between wavenumber and the loading coefficients of the first three principal components is shown in Fig. 3. The contribution rates of the first three principal components reached 96\%, which could explain most information from the original data. Therefore, the feature spectra were selected according to the changes between the maximum and minimum

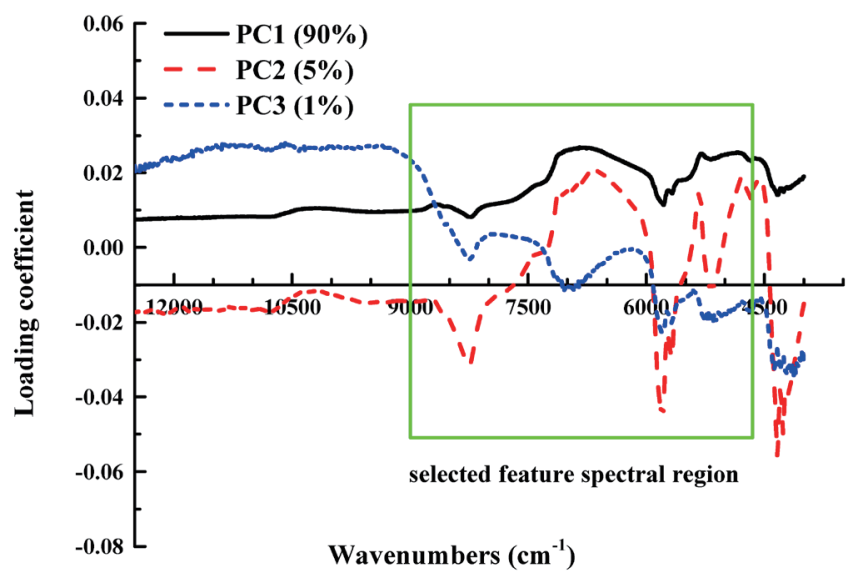

Fig. 3. (Color online) Plot of loading coefficient based on the prediction of glycogen content based on nontreated spectra in $12500-4000 \mathrm{~cm}^{-1}$ region. 
Table 3

Performance comparison of PLSR models for predicting glycogen and moisture contents based on feature spectral data preprocessed by different methods.

\begin{tabular}{|c|c|c|c|c|c|c|c|c|}
\hline & \multirow{2}{*}{ Preprocessing } & \multirow{2}{*}{$\begin{array}{l}\text { Spectral region } \\
\left(\mathrm{cm}^{-1}\right)\end{array}$} & \multirow{2}{*}{ LV } & \multicolumn{2}{|c|}{ Calibration } & \multicolumn{2}{|c|}{ Prediction } & \multirow{2}{*}{ RPD } \\
\hline & & & & $R_{c}^{2}$ & RMSEC & $R_{p}{ }^{2}$ & RMSEC & \\
\hline \multirow{6}{*}{$\begin{array}{l}\text { Glycogen } \\
(\mathrm{mg} / \mathrm{g})\end{array}$} & Nontreated & $9000-4300$ & 5 & 0.556 & 0.305 & 0.255 & 0.427 & 1.101 \\
\hline & Smoothing & $8800-4000$ & 4 & 0.471 & 0.333 & 0.174 & 0.431 & 1.090 \\
\hline & SNV & $9000-4300$ & 3 & 0.517 & 0.319 & 0.297 & 0.393 & 1.196 \\
\hline & MSC & $9000-4300$ & 2 & 0.459 & 0.337 & 0.277 & 0.393 & 1.196 \\
\hline & Smoothing-SNV & $9000-4300$ & 2 & 0.463 & 0.336 & 0.415 & 0.386 & 1.218 \\
\hline & Smoothing-MSC & $9000-4300$ & 2 & 0.459 & 0.337 & 0.349 & 0.399 & 1.178 \\
\hline \multirow{6}{*}{$\begin{array}{l}\text { Moisture } \\
(\mathrm{w} / \mathrm{w} \%)\end{array}$} & Nontreated & $8700-4300$ & 4 & 0.856 & 1.988 & 0.751 & 2.619 & 2.047 \\
\hline & Smoothing & $9900-4000$ & 4 & 0.838 & 2.116 & 0.725 & 2.846 & 1.883 \\
\hline & SNV & $8700-4300$ & 2 & 0.800 & 2.347 & 0.738 & 2.755 & 1.946 \\
\hline & MSC & $8700-4300$ & 3 & 0.826 & 2.188 & 0.750 & 2.737 & 1.958 \\
\hline & Smoothing-SNV & $8700-4300$ & 3 & 0.826 & 2.19 & 0.755 & 2.710 & 1.978 \\
\hline & Smoothing-MSC & $8700-4300$ & 3 & 0.826 & 2.188 & 0.750 & 2.746 & 1.952 \\
\hline
\end{tabular}

values of the loading coefficient. Furthermore, PLS analysis was carried out on the basis of the above selected spectra. As shown in Fig. 3, for nontreated spectra, the region between 9000 and $4300 \mathrm{~cm}^{-1}$ was selected as feature spectra for establishing new quantitative analysis models to predict glycogen content. The same analysis method was used for selecting the feature spectra from other spectra preprocessed by different methods. The performances of PLSR models for predicting glycogen and moisture contents based on feature spectra preprocessed by different methods are shown in Table 3.

For glycogen prediction, although not very satisfactory results were obtained, the best PLSR model based on feature spectra with the highest $R_{p}{ }^{2}$ value of 0.415 , the lowest RMSEP value of $0.386 \mathrm{mg} / \mathrm{g}$, and an RPD value of 1.218 was obtained. These results were similar to those obtained using the best PLSR model based on full spectra. By analyzing the moisture content, the performance characteristics of PLSR models based on full spectra were better than those of PLSR models based on feature spectra. Through the analysis results, although the above feature spectral selection method can remove spectral regions unrelated to glycogen and moisture contents and achieve data reduction, the performance characteristics of PLSR models based on full spectra were generally better than those of PLSR models based on feature spectra. Taken together, the PLSR model for predicting the glycogen content of Japanese wagyu beef samples could be further enhanced for better $R_{p}{ }^{2}$ and higher RPD values with more samples in the calibration set, and the PLSR model based on full spectra with smoothing-MSC treatment was implemented to predict the moisture content of Japanese wagyu beef samples in Tottori Prefecture, Japan.

\section{Conclusion}

In this study, the use of FT-NIR spectroscopy coupled with multivariable data processing to predict the glycogen and moisture contents in Japanese wagyu beef samples was investigated. 
The obtained results showed that the present work generated the PLSR models of moisture content with a prediction performance similar to that in a previous work, and the achieved predictive models were considered to be good. The performance of PLSR models for glycogen content prediction was not very strong; therefore, further studies are required to obtain more robust models for analytical purposes. However, FT-NIR is a promising method for the prediction of glycogen and moisture contents, which are considered to affect the palatability of Japanese wagyu beef samples.

\section{Acknowledgments}

We are grateful to the members of the Tottori office of JA Zen-Noh Meat Foods Co., Ltd. for the samples of Japanese wagyu beef. We also wish to express our gratitude to Dr. Masatoshi Kozaki and Mr. Chenxi Shen for their assistance in measurement and data analyses. In addition, this work was partly supported by the JST-Mirai Program under Grant Number JPMJMI18D8.

\section{References}

1 R. J. Polkinghorne, T. Nishimura, K. E. Neath, and R. Watson: J. Anim. Sci. 82 (2011) 325. https://doi. org/10.1111/j.1740-0929.2010.00825.x

2 Y. Ueda, A. Watanabe, M. Higuchi, H. Shingu, S. Kushibiki, and M. Shinoda: J. Anim. Sci. 78 (2007) 189. https://doi.org/10.1111/j.1740-0929.2007.00424.x

3 E. N. Bermingham, M. G. Reis, A. K. Subbaraj, D. Cameron-Smith, K. Fraser, A. Jonker, and C. R. Craigie: Meat Sci. 140 (2018) 26. https://doi.org/10.1016/J.MEATSCI.2018.02.012

4 T. Gotoh, H. Takahashi, T. Nishimura, K. Kuchida, and H. Mannen: Anim. Front. 4 (2014) 46. https://doi. org/10.2527/af.2014-0033

5 M. Motoyama, K. Sasaki, and A. Watanabe: MESC. 120 (2016) 10. https://doi.org/10.1016/j.meatsci.2016.04.026

6 M. Matsuishi, J. Kume, Y. Itou, M. Takahashi, M. Arai, H. Nagatomi, K. Watanabe, F. Hayase, and A. Okitani: Nihon Chikusan Gakkaiho. 75 (2004) 409. https://doi.org/10.2508/chikusan.75.409

7 Y. Han, X. Wang, Y. Cai, Z. Li, L. Zhao, H. Wang, J. Jin, Y. Cai, L. Xu, and L. Zhu: Meat Sci. 129 (2017) 38. https://doi.org/10.1016/j.meatsci.2017.02.016

8 M. F. Miller, M. A. Carr, C. B. Ramsey, K. L. Crockett, and L. C. Hoover: J. Anim. Sci. 79 (2001) 3062. https://doi.org/10.2527/2001.79123062x

9 J. W. Savell, C. L. Lorenzen, T. R. Neely, R. K. Miller, J. D. Tatum, J. W. Wise, J. F. Taylor, M. J. Buyck, and J. O. Reagan: J. Anim. Sci. 77 (1999) 645. https://doi.org/10.2527/1999.773645x

10 C. H. Corbin, T. G. O'Quinn, A. J. Garmyn, J. F. Legako, M. R. Hunt, T. T. N. Dinh, R. J. Rathmann, J. C. Brooks, and M. F. Miller: Meat Sci. 100 (2015) 24. https://doi.org/10.1016/J.MEATSCI.2014.09.009

11 T. G. O'Quinn, J. F. Legako, J. C. Brooks, and M. F. Miller: Transl. Anim. Sci. 2 (2018) 26. https://doi. org/10.1093/tas/txx008

12 J. M. Behrends, K. J. Goodson, M. Koohmaraie, S. D. Shackelford, T. L. Wheeler, W. W. Morgan, J. O. Reagan, B. L. Gwartney, J. W. Wise, and J. W. Savell: J. Anim. Sci. 83 (2005) 2869. https://doi.org/10.2527/2005.83122869x

13 J. M. Behrends, K. J. Goodson, M. Koohmaraie, S. D. Shackelford, T. L. Wheeler, W. W. Morgan, J. O. Reagan, B. L. Gwartney, J. W. Wise, and J. W. Savell: J. Anim. Sci. 83 (2005) 662. https://doi.org/10.2527/2005.833662x

14 T. G. O'Quinn, J. C. Brooks, R. J. Polkinghorne, A. J. Garmyn, B. J. Johnson, J. D. Starkey, R. J. Rathmann, and M. F. Miller: J. Anim. Sci. 90 (2012) 626. https://doi.org/10.2527/jas.2011-4282

15 L. W. Lucherk, T. G. O'Quinn, J. F. Legako, R. J. Rathmann, J. C. Brooks, and M. F. Miller: Meat Sci. 122 (2016) 145. https://doi.org/10.1016/j.meatsci.2016.08.005

16 T. Oe, M. Toriyama, T. Okagaki: Proc. 121st Japanese Society of Animal Science Conf. (2016) 55. (In Japanese).

17 A. Hargreaves B, L. Barrales V, D. Barrales Z, J. L. Riveros F, and I. Peña R: Chil. J. Agric. Res. 69 (2009) 366. https://doi.org/10.4067/S0718-58392009000300009 
18 R. W. Purchas and R. Aungsupakorn: Meat Sci. 34 (1993) 163. https://doi.org/10.1016/0309-1740(93)90025-D

19 J. A. Silva, L. Patarata, and C. Martins: Meat Sci. 52 (1999) 453. https://doi.org/10.1016/S0309-1740(99)000297

20 T. Komatsu, N. Shoji, K. Saito, and K. Suzuki: J. Anim. Sci. 85 (2014) 793. https://doi.org/10.1111/asj.12201

21 M. Kamruzzaman, Y. Makino, and S. Oshita: Food Chem. 196 (2016) 1084. https://doi.org/10.1016/ J.FOODCHEM.2015.10.051

22 J. Weeranantanaphan, G. Downey, P. Allen, and D. W. Sun: J. Near Infrared Spectrosc. 19 (2011) 61. https:// doi.org/10.1255/jnirs.924

23 Y. Dixit, M. P. Casado-Gavalda, R. Cama-Moncunill, X. Cama-Moncunill, M. Markiewicz-Keszycka, P. J. Cullen, and C. Sullivan: Compr. Rev. Food Sci. Food Saf. 16 (2017) 1172. https://doi.org/10.1111/15414337.12295

24 L. Q. Hu, C. L. Yin, and Z. P. Zeng: Spectrochim. Acta, Part A 151 (2015) 34. https://doi.org/10.1016/ j.saa.2015.06.067

25 N. Morsy and D. W. Sun: Meat Sci. 93 (2013) 292. https://doi.org/10.1016/j.meatsci.2012.09.005

26 Y. Z. Feng and D. W. Sun: Talanta 105 (2013) 244. https://doi.org/10.1016/j.talanta.2012.11.042

27 V. S. Kodogiannis, I. Petrounias, and J. Lygouras: Adv. Intell. Syst. Comput. 323 (2015) 303. https://doi. org/10.1007/978-3-319-11310-4_26

28 Y. Dixit, M. P. Casado-Gavalda, R. Cama-Moncunill, X. Cama-Moncunill, F. Jacoby, P. J. Cullen, and C. Sullivan: J. Food Eng. 175 (2016) 58. https://doi.org/10.1016/j.jfoodeng.2015.12.004

29 M. De Marchi: Meat Sci. 94 (2013) 455. https://doi.org/10.1016/j.meatsci.2013.03.003

30 K. I. Kobayashi, Y. Matsui, Y. Maebuchi, T. Toyota, and S. Nakauchi: J. Near Infrared Spectrosc. 18 (2010) 301. https://doi.org/10.1255/jnirs. 896

31 S. Piao, T. Okura, and M. Irie: Meat Sci. 137 (2018) 258. https://doi.org/10.1016/J.MEATSCI.2017.11.032

32 K. Suzuki, H. Shioura, S. Yokota, K. Katoh, S. G. Roh, F. Iida, T. Komatsu, N. Syoji, H. Sakuma, and S. Yamada: J. Anim. Sci. 88 (2017) 421. https://doi.org/10.1111/asj.12663

33 Japan Meat Grading Association: http://www.jmga.or.jp/ (accessed January 2019).

34 C. E. Dreiling, D. E. Brown, L. Casale, and L. Kelly: Meat Sci. 20 (1987) 167. https://doi.org/10.1016/03091740(87)90009-X

35 M. Vidal and J. M. Amigo: Chemom. Intell. Lab. Syst. 117 (2012) 138. https://doi.org/10.1016/ j.chemolab.2012.05.009

36 X. Huang, H. Xu, L. Wu, H. Dai, L. Yao, and F. Han: Anal. Methods. 8 (2016) 2929. https://doi.org/10.1039/ c5ay03005f

37 D. F. Barbin, C. M. Kaminishikawahara, A. L. Soares, I. Y. Mizubuti, M. Grespan, M. Shimokomaki, and E. Y. Hirooka: Food Chem. 168 (2015) 554. https://doi.org/10.1016/j.foodchem.2014.07.101

38 C. Esquerre, A. A. Gowen, G. Downey, and C. P. O’Donnell: J. Near Infrared Spectrosc. 20 (2012) 537. https:// doi.org/10.1255/jnirs.1014

39 M. De Marchi, C. L. Manuelian, S. Ton, D. Manfrin, M. Meneghesso, M. Cassandro, and M. Penasa: Meat Sci. 125 (2017) 61. https://doi.org/10.1016/j.meatsci.2016.11.014

40 W. Saeys, A. M. Mouazen, and H. Ramon: Biosyst. Eng. 91 (2005) 393. https://doi.org/10.1016/ J.BIOSYSTEMSENG.2005.05.001

41 J. F. Hocquette, R. Botreau, B. Picard, A. Jacquet, D. W. Pethick, and N. D. Scollan: Meat Sci. 92 (2012) 197. https://doi.org/10.1016/j.meatsci.2012.04.007

42 W. Przybylski, B. Sionek, D. Jaworska, and V. Santé-Lhoutellier: Meat Sci. 117 (2016) 7. https://doi. org/10.1016/j.meatsci.2016.02.025

43 D. W. Pethick, J. B. Rowe, and G. Tudor: Recent Adv. Anim. Nutr. Aust. 7 (1995) 97.

44 K. Sakai, K. Shimba, K. Kotani, and Y. Jimbo: Sens. Mater. 30 (2018) 2979. https://doi.org/10.18494/ SAM.2018.2051

45 M. Cunningham, M. R. Brown, P. D. Kube, S. O’Connor, and H. King: J. Shellfish Res. 31 (2013) 1051. https:// doi.org/10.2983/035.031.0417

46 M. R. Brown: Aquaculture 317 (2011) 233. https://doi.org/10.1016/j.aquaculture.2011.04.017

47 D. Lomiwes, M. M. Reis, E. Wiklund, O. A. Young, and M. North: Meat Sci. 86 (2010) 999. https://doi. org/10.1016/j.meatsci.2010.08.007

48 K. Rosenvold, E. Micklander, P. W. Hansen, R. Burling-Claridge, M. Challies, C. Devine, and M. North: Meat Sci. 82 (2009) 379. https://doi.org/10.1016/j.meatsci.2009.02.010 